\title{
ON THE SCATTERING OF ELECTROMAGNETIC WAVES BY BI-ISOTROPIC SPHERICAL SHELL
}

\begin{abstract}
In this article an analytical solution of the problem of scattering of electromagnetic waves on bi-isotropic spherical shell has been presented. Secondary electromagnetic fields are introduced as a superposition of spherical vector wave functions. The solution of the boundary problem is reduced to solving a system of linear algebraic equations for the coefficients which are included in the presentation of the secondary fields. The formula to calculate the directional diagram of electric field in the far field and numerical results for different values of parameters have been obtained. The results may find practical application in the development and design of electromagnetic screens.
\end{abstract}

Keywords: bi-isotropic spherical shell, electromagnetic waves, spherical vector wave functions, directional diagram of electric field

\section{Introduction}

Within electrodynamics the interest to the study of complex electromagnetic medium has increased in the mid-80s of the XX century. The chiral medium is an example of such a medium that is a set of conductive mirror-asymmetric microelements in the isotropic magnetoelectric medium. In [1-3] the detailed classification of chiral media is described. Bi-isotropic media are a generalization of chiral media. Besides chirality, these media also have the property of non-reciprocity [4-6].

The interest to the study of the scattering of electromagnetic waves on biisotropic media is due to the ability of these media to both enhance and absorb electromagnetic fields [8-10].

We will consider a few scientific works related to this topic. In [11] the study of the chirality influence of the medium on the electric dipole electromag-

\footnotetext{
1 Autor do korespondencji/corresponding author: Gennady Shushkevich, Yanka Kupala State University of Grodno, 22, Ozheshko St., 230023 Grodno, Belarus, e-mail: g_shu@ @ut.by

2 Svetlana Shushkevich, Yanka Kupala State University of Grodno, e-mail: spusha@list.ru

${ }^{3}$ Aleksandr Kuts, Yanka Kupala State University of Grodno, e-mail: sadako1983@mail.ru
} 
netic field is presented. An analytical solution of the diffraction problem of a plane electromagnetic wave on the bi-isotropic ball is proposed in [12,13]. An analytical solution of the plane electromagnetic wave diffraction problem on the plane composite layer was considered in [14]. In [15] the electromagnetic fields penetration of electric and magnetic dipoles through plane bi-isotropic layer is described. The electromagnetic waves reflection from the plane chiral structures is studied in $[16,17]$. In [18] a problem of the plane electromagnetic wave scattering on a metal cylinder covered with a chiral layer is solved using the partialarea method.

In this work an analytical axisymmetric solution of the scattering electromagnetic field of the electric and magnetic dipoles on the bi-isotropic spherical shell problem has been built. The computational experiment with some geometric parameters of the problem and different electromagnetic parameters of a biisotropic material spherical shell has been conducted.

\section{Problem formulation}

Let the whole space $R^{3}$ be divided by two spheres $S_{1}$ and $S_{2}$ into three areas $D_{0}, D_{1}, D_{2}$ (fig. 1). The two areas $D_{0}, D_{2}$ are filled with homogeneous isotropic medium with a permittivity $\varepsilon_{0}$ and magnetic permeability $\mu_{0}$, area $D_{1}$ is filled with homogeneous bi-isotropic medium, which is characterized by the material parameters $\varepsilon, \mu, G, Z$. The inner radius of the spherical area $D_{1}$ is equal $a_{1}$ and the outer radius is equal $a_{2}$. The point $\mathrm{O}_{1}$ is the source of the electromagnetic field consisting of both electric and magnetic dipoles. The distance between the points $\mathrm{O}, \mathrm{O}_{1}$ is denoted by $\mathrm{h}$. We assume that on the two surfaces $\mathrm{S}_{1}, \mathrm{~S}_{2}$ there are no surface currents and charges.

Let denote strength vectors of electric and magnetic fields of the initial electromagnetic field as $\vec{E}_{d}, \vec{H}_{d}$. As a result of the interaction of the initial electromagnetic field with the bi-isotropic spherical shell $\mathrm{D}_{1}$ secondary fields are being formed. Let $\vec{E}_{0}, \vec{H}_{0}$ be the secondary field in the area of $D_{0} ; \vec{E}_{1}, \vec{H}_{1}$ be the secondary field in the area $D_{1} ; \vec{E}_{2}, \vec{H}_{2}$ be the secondary field in the area $D_{2}$.

The real electromagnetic fields are determined by the formulas:

$\overrightarrow{\mathbb{E}}_{\mathrm{j}}=\operatorname{Re}\left(\overrightarrow{\mathrm{E}}_{\mathrm{j}} \mathrm{e}^{-\mathrm{i} \omega \mathrm{t}}\right), \quad \overrightarrow{\mathbb{H}}_{\mathrm{j}}=\operatorname{Re}\left(\overrightarrow{\mathrm{H}}_{\mathrm{j}} \mathrm{e}^{-\mathrm{i} \omega \mathrm{t}}\right), \mathrm{j}=0,1,2 ; \mathrm{i}$ is an imaginary unit.

The secondary electromagnetic fields should be defined $\overrightarrow{\mathrm{E}}_{0}, \overrightarrow{\mathrm{H}}_{0} \in \mathrm{C}^{1}\left(\mathrm{D}_{0}\right) \cap \mathrm{C}\left(\overline{\mathrm{D}}_{0}\right), \overrightarrow{\mathrm{E}}_{1}, \overrightarrow{\mathrm{H}}_{1} \in \mathrm{C}\left(\mathrm{D}_{1}\right) \cap \mathrm{C}\left(\overline{\mathrm{D}}_{1}\right), \overrightarrow{\mathrm{E}}_{2}, \overrightarrow{\mathrm{H}}_{2} \in \mathrm{C}\left(\mathrm{D}_{2}\right) \cap \mathrm{C}\left(\overline{\mathrm{D}}_{2}\right)$ that satisfy

- Maxwell's equations [19-21]:

$$
\operatorname{rot} \overrightarrow{\mathrm{E}}_{\mathrm{j}}=\mathrm{i} \omega \mu_{0} \overrightarrow{\mathrm{H}}_{\mathrm{j}}, \quad \operatorname{rot} \overrightarrow{\mathrm{H}}_{\mathrm{j}}=-\mathrm{i} \omega \varepsilon_{0} \overrightarrow{\mathrm{E}}_{\mathrm{j}}, \quad \mathrm{j}=0,2
$$




$$
\operatorname{rot} \overrightarrow{\mathrm{E}}_{1}=i \omega\left(\mu \overrightarrow{\mathrm{H}}_{1}+\mathrm{Z} \overrightarrow{\mathrm{E}}_{1}\right), \quad \operatorname{rot} \overrightarrow{\mathrm{H}}_{1}=-\mathrm{i} \omega\left(\varepsilon \overrightarrow{\mathrm{E}}_{1}+\mathrm{GH}_{1}\right)
$$

where $G=(\tau+i \kappa) \sqrt{\varepsilon_{0} \mu_{0}}, Z=(\tau-i \kappa) \sqrt{\varepsilon_{0} \mu_{0}}, \kappa$ is chirality parameter, $\tau$ is the Tellegen parameter,

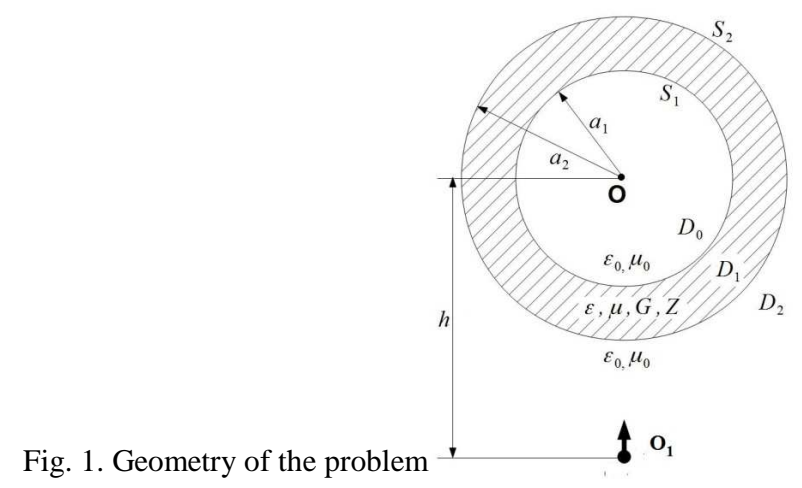

- boundary conditions on surfaces $\mathrm{S}_{1}, \mathrm{~S}_{2}$ :

$$
\begin{aligned}
& {\left.\left[\overrightarrow{\mathrm{e}}_{\mathrm{z}}, \overrightarrow{\mathrm{E}}_{\mathrm{d}}+\overrightarrow{\mathrm{E}}_{2}\right]\right|_{\mathrm{S}_{2}}=\left.\left[\overrightarrow{\mathrm{e}}_{\mathrm{z}}, \overrightarrow{\mathrm{E}}_{1}\right]\right|_{\mathrm{S}_{2}},\left.\quad\left[\overrightarrow{\mathrm{e}}_{\mathrm{z}}, \overrightarrow{\mathrm{H}}_{\mathrm{d}}+\overrightarrow{\mathrm{H}}_{2}\right]\right|_{\mathrm{S}_{2}}=\left.\left[\overrightarrow{\mathrm{e}}_{\mathrm{z}}, \overrightarrow{\mathrm{H}}_{1}\right]\right|_{\mathrm{S}_{2}},} \\
& {\left.\left[\overrightarrow{\mathrm{e}}_{\mathrm{z}}, \overrightarrow{\mathrm{E}}_{1}\right]\right|_{\mathrm{S}_{1}}=\left.\left[\overrightarrow{\mathrm{e}}_{\mathrm{z}}, \overrightarrow{\mathrm{E}}_{0}\right]\right|_{\mathrm{S}_{1}},\left.\quad\left[\overrightarrow{\mathrm{e}}_{\mathrm{z}}, \overrightarrow{\mathrm{H}}_{1}\right]\right|_{\mathrm{S}_{1}}=\left.\left[\overrightarrow{\mathrm{e}}_{\mathrm{z}}, \overrightarrow{\mathrm{H}}_{0}\right]\right|_{\mathrm{S}_{1}},}
\end{aligned}
$$

and the radiation condition at infinity [19]

$$
\lim _{r \rightarrow \infty} r\left(\frac{\partial \overrightarrow{\mathrm{E}}_{2}}{\partial \mathrm{r}}-\mathrm{ik}_{0} \overrightarrow{\mathrm{E}}_{2}\right)=0, \quad \lim _{\mathrm{r} \rightarrow \infty} \mathrm{r}\left(\frac{\partial \overrightarrow{\mathrm{H}}_{2}}{\partial \mathrm{r}}-\mathrm{ik}_{0} \overrightarrow{\mathrm{H}}_{2}\right)=0,
$$

where $\mathrm{k}_{0}=\omega \sqrt{\varepsilon_{0} \mu_{0}}$ is a wave number.

\section{Presentation of problem solution}

The initial electromagnetic field $\overrightarrow{\mathrm{E}}_{\mathrm{d}}, \overrightarrow{\mathrm{H}}_{\mathrm{d}}$ will be presented through the wave vector spherical functions $[20,21]$ :

$$
\left.\begin{array}{l}
\overrightarrow{\mathrm{E}}_{\mathrm{d}}=\mathrm{E}_{0}^{(\mathrm{e})} \tilde{\overrightarrow{\mathrm{n}}}_{01}\left(\mathrm{r}_{1}, \theta_{1}, \mathrm{k}_{0}\right)+\mathrm{E}_{0}^{(\mathrm{m})} \tilde{\overrightarrow{\mathrm{m}}}_{01}\left(\mathrm{r}_{1}, \theta_{1}, \mathrm{k}_{0}\right), \\
\overrightarrow{\mathrm{H}}_{\mathrm{d}}=\mathrm{H}_{0}^{(\mathrm{m})} \tilde{\overrightarrow{\mathrm{n}}}_{01}\left(\mathrm{r}_{1}, \theta_{1}, \mathrm{k}_{0}\right)+\mathrm{H}_{0}^{(\mathrm{e})} \tilde{\overrightarrow{\mathrm{m}}}_{01}\left(\mathrm{r}_{1}, \theta_{1}, \mathrm{k}_{0}\right),
\end{array}\right\},
$$


where $\mathrm{H}_{0}^{(\mathrm{e})}=\frac{\mathrm{k}_{0}}{\mathrm{i} \omega \mu_{0}} \mathrm{E}_{0}^{(\mathrm{e})}, \mathrm{H}_{0}^{(\mathrm{m})}=\frac{\mathrm{k}_{0}}{\mathrm{i} \omega \mu_{0}} \mathrm{E}_{0}^{(\mathrm{m})}, \mathrm{E}_{0}^{(\mathrm{e})}, \mathrm{E}_{0}^{(\mathrm{m})}$ are the definite constants,

$$
\left.\begin{array}{l}
\tilde{\overrightarrow{\mathrm{n}}}_{0 \mathrm{n}}(\mathrm{r}, \theta, \mathrm{k})=\frac{\mathrm{n}(\mathrm{n}+1)}{\mathrm{kr}} \mathrm{h}_{\mathrm{n}}^{(1)}(\mathrm{kr}) \mathrm{P}_{\mathrm{n}}(\cos \theta) \overrightarrow{\mathrm{e}}_{\mathrm{r}}+\mathrm{g}_{\mathrm{n}}^{(1)}(\mathrm{kr}) \mathrm{P}_{\mathrm{n}}^{1}(\cos \theta) \overrightarrow{\mathrm{e}}_{\theta}, \\
\tilde{\overrightarrow{\mathrm{m}}}_{0 \mathrm{n}}(\mathrm{r}, \theta, \mathrm{k})=-\mathrm{h}_{\mathrm{n}}^{(1)}(\mathrm{kr}) \mathrm{P}_{\mathrm{n}}^{1}(\cos \theta) \overrightarrow{\mathrm{e}}_{\varphi}, \\
\mathrm{g}_{\mathrm{n}}^{(1)}(\mathrm{x})=\frac{1}{\mathrm{x}} \frac{\mathrm{d}}{\mathrm{dx}}\left(\mathrm{xh}_{\mathrm{n}}^{(1)}(\mathrm{x})\right)=\frac{1}{2 \mathrm{n}+1}\left((\mathrm{n}+1) \mathrm{h}_{\mathrm{n}-1}^{(1)}(\mathrm{x})-\mathrm{nh}_{\mathrm{n}+1}^{(1)}(\mathrm{x})\right),
\end{array}\right\}
$$

$P_{n}(x)$ are the Legendre polynomials, $P_{n}^{1}(\cos \theta)$ are the associated Legendre functions of the first kind, $h_{n}^{(1)}(x)$ are the spherical Hankel functions of the first kind [22].

The secondary electromagnetic field in the area of $\mathrm{D}_{0}$ will be presented as a superposition of vector spherical wave functions which satisfies equations (1):

$$
\left.\begin{array}{l}
\overrightarrow{\mathrm{E}}_{0}=\mathrm{E}_{0} \sum_{\mathrm{n}=1}^{\infty}\left[\mathrm{a}_{\mathrm{n}}^{(1)} \overrightarrow{\mathrm{m}}_{0 \mathrm{n}}\left(\mathrm{r}, \theta, \mathrm{k}_{0}\right)+\mathrm{a}_{\mathrm{n}}^{(2)} \overrightarrow{\mathrm{n}}_{0 \mathrm{n}}\left(\mathrm{r}, \theta, \mathrm{k}_{0}\right)\right], \\
\overrightarrow{\mathrm{H}}_{0}=\mathrm{H}_{0} \sum_{\mathrm{n}=1}^{\infty}\left[\mathrm{a}_{\mathrm{n}}^{(1)} \overrightarrow{\mathrm{n}}_{0 \mathrm{n}}\left(\mathrm{r}, \theta, \mathrm{k}_{0}\right)+\mathrm{a}_{\mathrm{n}}^{(2)} \overrightarrow{\mathrm{m}}_{0 \mathrm{n}}\left(\mathrm{r}, \theta, \mathrm{k}_{0}\right)\right],
\end{array}\right\}
$$

where

$$
\left.\begin{array}{l}
\overrightarrow{\mathrm{n}}_{0 \mathrm{n}}(\mathrm{r}, \theta, \mathrm{k})=\frac{\mathrm{n}(\mathrm{n}+1)}{\mathrm{kr}} \mathrm{j}_{\mathrm{n}}(\mathrm{kr}) \mathrm{P}_{\mathrm{n}}(\cos \theta) \overrightarrow{\mathrm{e}}_{\mathrm{r}}+\mathrm{g}_{\mathrm{n}}(\mathrm{kr}) \mathrm{P}_{\mathrm{n}}^{1}(\cos \theta) \overrightarrow{\mathrm{e}}_{\theta}, \\
\overrightarrow{\mathrm{m}}_{0 \mathrm{n}}(\mathrm{r}, \theta, \mathrm{k})=-\mathrm{j}_{\mathrm{n}}(\mathrm{kr}) \mathrm{P}_{\mathrm{n}}^{1}(\cos \theta) \overrightarrow{\mathrm{e}}_{\varphi}, \\
\mathrm{g}_{\mathrm{n}}(\mathrm{x})=\frac{1}{\mathrm{x}} \frac{\mathrm{d}}{\mathrm{d} \mathrm{x}}\left(\mathrm{xj}_{\mathrm{n}}(\mathrm{x})\right)=\frac{1}{2 \mathrm{n}+1}\left((\mathrm{n}+1) \mathrm{j}_{\mathrm{n}-1}(\mathrm{x})-\mathrm{nj}_{\mathrm{n}+1}(\mathrm{x})\right),
\end{array}\right\}
$$

$\mathrm{E}_{0}, \mathrm{H}_{0}$ are the definite constants, $\mathrm{j}_{\mathrm{n}}(\mathrm{x})$ are the spherical Bessel functions of the first kind [22].

The secondary electromagnetic field in the area of $\mathrm{D}_{2}$ will be presented as a superposition of vector spherical wave functions, which satisfies the equations (2) and at infinity the condition (5): 


$$
\left.\begin{array}{l}
\overrightarrow{\mathrm{E}}_{2}=\mathrm{E}_{0} \sum_{\mathrm{n}=1}^{\infty}\left[\mathrm{d}_{\mathrm{n}}^{(1)} \tilde{\overrightarrow{\mathrm{m}}}_{0 \mathrm{n}}\left(\mathrm{r}, \theta, \mathrm{k}_{0}\right)+\mathrm{d}_{\mathrm{n}}^{(2)} \tilde{\overrightarrow{\mathrm{n}}}_{0 \mathrm{n}}\left(\mathrm{r}, \theta, \mathrm{k}_{0}\right)\right], \\
\overrightarrow{\mathrm{H}}_{2}=\mathrm{H}_{0} \sum_{\mathrm{n}=1}^{\infty}\left[\mathrm{d}_{\mathrm{n}}^{(1)} \tilde{\overrightarrow{\mathrm{n}}}_{0 \mathrm{n}}\left(\mathrm{r}, \theta, \mathrm{k}_{0}\right)+\mathrm{d}_{\mathrm{n}}^{(2)} \tilde{\overrightarrow{\mathrm{m}}}_{0 \mathrm{n}}\left(\mathrm{r}, \theta, \mathrm{k}_{0}\right)\right],
\end{array}\right\}
$$

The secondary electromagnetic field in the area of $\mathrm{D}_{1}$ will be expressed as a superposition of spherical vector wave functions in the composite media [21], which satisfies the equations (2):

$$
\overrightarrow{\mathrm{E}}_{1}=\overrightarrow{\mathrm{E}}_{1}^{(+)}+\overrightarrow{\mathrm{E}}_{1}^{(-)}, \quad \overrightarrow{\mathrm{H}}_{1}=\overrightarrow{\mathrm{H}}_{1}^{(+)}+\overrightarrow{\mathrm{H}}_{1}^{(-)}
$$

where

$$
\begin{aligned}
& \left.\begin{array}{l}
\overrightarrow{\mathrm{E}}_{1}^{+}=\mathrm{E}_{0} \sum_{\mathrm{n}=1}^{\infty}\left[\mathrm{b}_{\mathrm{n}}^{(1)} \tilde{\overrightarrow{\mathrm{K}}}_{0 \mathrm{n}}^{(1)}\left(\mathrm{r}, \theta, \mathrm{k}_{1}\right)+\mathrm{b}_{\mathrm{n}}^{(2)} \tilde{\overrightarrow{\mathrm{K}}}_{0 \mathrm{n}}^{(2)}\left(\mathrm{r}, \theta, \mathrm{k}_{2}\right)\right], \\
\overrightarrow{\mathrm{H}}_{1}^{+}=\mathrm{E}_{0} \sum_{\mathrm{n}=1}^{\infty}\left[\mathrm{b}_{\mathrm{n}}^{(1)} \mathrm{p}_{1} \tilde{\overline{\mathrm{K}}}_{0 \mathrm{n}}^{(1)}\left(\mathrm{r}, \theta, \mathrm{k}_{1}\right)+\mathrm{b}_{\mathrm{n}}^{(2)} \mathrm{p}_{2} \tilde{\overline{\mathrm{K}}}_{0 \mathrm{n}}^{(2)}\left(\mathrm{r}, \theta, \mathrm{k}_{2}\right)\right],
\end{array}\right\} \\
& \overrightarrow{\mathrm{E}}_{1}^{-}=\mathrm{E}_{0} \sum_{\mathrm{n}=1}^{\infty}\left[\mathrm{c}_{\mathrm{n}}^{(1)} \overrightarrow{\mathrm{K}}_{0 \mathrm{n}}^{(1)}\left(\mathrm{r}, \theta, \mathrm{k}_{1}\right)+\mathrm{c}_{\mathrm{n}}^{(2)} \overrightarrow{\mathrm{K}}_{0 \mathrm{n}}^{(2)}\left(\mathrm{r}, \theta, \mathrm{k}_{2}\right)\right] \\
& \overrightarrow{\mathrm{H}}_{1}^{-}=\mathrm{E}_{0} \sum_{\mathrm{n}=1}^{\infty}\left[\mathrm{c}_{\mathrm{n}}^{(1)} \mathrm{p}_{1} \overrightarrow{\mathrm{K}}_{0 \mathrm{n}}^{(1)}\left(\mathrm{r}, \theta, \mathrm{k}_{1}\right)+\mathrm{c}_{\mathrm{n}}^{(2)} \mathrm{p}_{2} \overrightarrow{\mathrm{K}}_{0 \mathrm{n}}^{(2)}\left(\mathrm{r}, \theta, \mathrm{k}_{2}\right)\right], \\
& \overrightarrow{\mathrm{K}}_{0 \mathrm{n}}^{(\mathrm{j})}\left(\mathrm{r}, \theta, \mathrm{k}_{\mathrm{j}}\right)=\overrightarrow{\mathrm{n}}_{0 \mathrm{n}}\left(\mathrm{r}, \theta, \mathrm{k}_{\mathrm{j}}\right)-\mathrm{q}_{\mathrm{j}} \overrightarrow{\mathrm{m}}_{0 \mathrm{n}}\left(\mathrm{r}, \theta, \mathrm{k}_{\mathrm{j}}\right), \\
& \tilde{\overrightarrow{\mathrm{K}}}_{0 \mathrm{n}}^{(\mathrm{j})}\left(\mathrm{r}, \theta, \mathrm{k}_{\mathrm{j}}\right)=\tilde{\overrightarrow{\mathrm{n}}}_{0 \mathrm{n}}\left(\mathrm{r}, \theta, \mathrm{k}_{\mathrm{j}}\right)-\mathrm{q}_{\mathrm{j}} \tilde{\overrightarrow{\mathrm{m}}}_{0 \mathrm{n}}\left(\mathrm{r}, \theta, \mathrm{k}_{\mathrm{j}}\right) \text {, } \\
& \mathrm{k}_{\mathrm{j}}=\sqrt{\mathrm{g}+0,5 \mathrm{a}^{2}+\mathrm{af}_{\mathrm{j}}}, \quad 0 \leq \arg \mathrm{k}_{\mathrm{j}}<\pi, \mathrm{g}=\omega^{2}(\varepsilon \mu-\mathrm{ZG}), \mathrm{a}=\mathrm{i} \omega(\mathrm{G}-\mathrm{Z}), \\
& \mathrm{f}_{\mathrm{j}}=(-1)^{\mathrm{j}} \mathrm{f}_{0}, \mathrm{f}_{0}=\sqrt{\omega^{2} \varepsilon \mu-\mathrm{b}^{2}}, \quad 0 \leq \arg \mathrm{f}_{0}<\pi \text {, } \\
& \mathrm{b}=0,5 \omega(\mathrm{G}+\mathrm{Z}), \mathrm{q}_{\mathrm{j}}=\frac{\mathrm{g}}{\mathrm{k}_{\mathrm{j}} \mathrm{g}_{\mathrm{j}}}, \mathrm{g}_{\mathrm{j}}=\mathrm{f}_{\mathrm{j}}-0,5 \mathrm{a}, \mathrm{p}_{\mathrm{j}}=\frac{1}{\mu}\left(\mathrm{ig} /\left(\omega \mathrm{g}_{\mathrm{j}}\right)-\mathrm{Z}\right), \mathrm{j}=1,2 .
\end{aligned}
$$

The unknown coefficients in the presentation of the secondary fields will be determined from the boundary conditions. 


\section{Fulfilment of boundary conditions}

First, we will express the function $\vec{E}_{d}, \vec{H}_{d}$ through the spherical vector wave functions in the coordinate system with the origin at the point $\mathrm{O}$ using the formula $[20,21]$ :

$$
\begin{aligned}
& \tilde{\overrightarrow{\mathrm{n}}}_{0 \mathrm{n}}\left(\mathrm{r}_{1}, \theta_{1}, \mathrm{k}_{0}\right)=\sum_{\mathrm{s}=1}^{\infty} \mathrm{A}_{\mathrm{s}}^{\mathrm{n}}\left(\mathrm{k}_{0} \mathrm{~h}, 0\right) \overrightarrow{\mathrm{n}}_{0 \mathrm{~s}}\left(\mathrm{r}, \theta, \mathrm{k}_{0}\right), 0 \leq \mathrm{r}<\mathrm{h}, \\
& \tilde{\overrightarrow{\mathrm{m}}}_{0 \mathrm{n}}\left(\mathrm{r}_{1}, \theta_{1}, \mathrm{k}_{0}\right)=\sum_{\mathrm{s}=1}^{\infty} \mathrm{A}_{\mathrm{s}}^{\mathrm{n}}\left(\mathrm{k}_{0} \mathrm{~h}, 0\right) \overrightarrow{\mathrm{m}}_{0 \mathrm{~s}}\left(\mathrm{r}, \theta, \mathrm{k}_{0}\right), 0 \leq \mathrm{r}<\mathrm{h},
\end{aligned}
$$

where

$$
\left.\begin{array}{l}
\mathrm{A}_{s}^{\mathrm{n}}\left(\mathrm{k}_{0} \mathrm{~h}, 0\right)=\mathrm{k}_{0} \mathrm{~h}\left[\frac{1}{(2 \mathrm{~s}+3)} \tilde{\mathrm{C}}_{\mathrm{s}+1}^{\mathrm{n}}+\frac{1}{(2 \mathrm{~s}-1)} \tilde{\mathrm{C}}_{\mathrm{s}-1}^{\mathrm{n}}\right]+\tilde{\mathrm{C}}_{\mathrm{s}}^{\mathrm{n}}, \\
\tilde{\mathrm{C}}_{\mathrm{s}}^{\mathrm{n}}=(2 \mathrm{~s}+1) \sum_{\sigma=|\mathrm{s}-\mathrm{n}|}^{\mathrm{s}+\mathrm{n}} \mathrm{i}^{\sigma+\mathrm{s}-\mathrm{n}} \mathrm{b}_{\sigma}^{(\mathrm{n} 0 \mathrm{~s} 0)} \mathrm{h}_{\sigma}^{(1)}\left(\mathrm{k}_{0} \mathrm{~h}\right) \mathrm{P}_{\sigma}(\cos \alpha),
\end{array}\right\}
$$

$\mathrm{b}_{\sigma}^{(\mathrm{n} 0 \mathrm{q} 0)}=(\mathrm{nq} 00 \mid \sigma 0)^{2}, \quad(\mathrm{nq} 00 \mid \sigma 0)$ are the Clebsch-Gordan coefficients [19], then

$$
\begin{aligned}
& \overrightarrow{\mathrm{E}}_{\mathrm{d}}=\mathrm{E}_{0}^{(\mathrm{e})} \sum_{\mathrm{n}=1}^{\infty} \mathrm{A}_{\mathrm{n}}^{1}\left(\mathrm{k}_{0} \mathrm{~h}, 0\right) \overrightarrow{\mathrm{n}}_{0 \mathrm{n}}\left(\mathrm{r}, \theta, \mathrm{k}_{0}\right)+\mathrm{E}_{0}^{(\mathrm{m})} \sum_{\mathrm{n}=1}^{\infty} \mathrm{A}_{\mathrm{n}}^{1}\left(\mathrm{k}_{0} \mathrm{~h}, 0\right) \overrightarrow{\mathrm{m}}_{0 \mathrm{n}}\left(\mathrm{r}, \theta, \mathrm{k}_{0}\right), \\
& \overrightarrow{\mathrm{H}}_{\mathrm{d}}=\mathrm{H}_{0}^{(\mathrm{m})} \sum_{\mathrm{n}=1}^{\infty} \mathrm{A}_{\mathrm{n}}^{1}\left(\mathrm{k}_{0} \mathrm{~h}, 0\right) \overrightarrow{\mathrm{n}}_{0 \mathrm{n}}\left(\mathrm{r}, \theta, \mathrm{k}_{0}\right)+\mathrm{H}_{0}^{(\mathrm{e})} \sum_{\mathrm{n}=1}^{\infty} \mathrm{A}_{\mathrm{n}}^{1}\left(\mathrm{k}_{0} \mathrm{~h}, 0\right) \overrightarrow{\mathrm{m}}_{0 \mathrm{n}}\left(\mathrm{r}, \theta, \mathrm{k}_{0}\right) .
\end{aligned}
$$

Taking into account (8), (10)-(13), (18), (19) and the condition of orthogonality of the associated Legendre functions on the interval $[0, \pi]$ the boundary conditions (3), (4) will become:

$$
\mathrm{M}(\mathrm{n}) \cdot \mathrm{V}(\mathrm{n})=\mathrm{F}(\mathrm{n}),
$$

where 


$$
\mathrm{M}(\mathrm{n})=\left(\begin{array}{cccccccc}
0 & \mathrm{~m}_{12} & \mathrm{~m}_{13} & \mathrm{~m}_{14} & \mathrm{~m}_{15} & \mathrm{~m}_{16} & 0 & 0 \\
\mathrm{~m}_{21} & 0 & \mathrm{~m}_{23} & \mathrm{~m}_{24} & \mathrm{~m}_{25} & \mathrm{~m}_{26} & 0 & 0 \\
\mathrm{~m}_{31} & 0 & \mathrm{~m}_{33} & \mathrm{~m}_{34} & \mathrm{~m}_{35} & \mathrm{~m}_{36} & 0 & 0 \\
0 & \mathrm{~m}_{42} & \mathrm{~m}_{43} & \mathrm{~m}_{44} & \mathrm{~m}_{45} & \mathrm{~m}_{46} & 0 & 0 \\
0 & 0 & \mathrm{~m}_{53} & \mathrm{~m}_{54} & \mathrm{~m}_{55} & \mathrm{~m}_{56} & 0 & \mathrm{~m}_{58} \\
0 & 0 & \mathrm{~m}_{63} & \mathrm{~m}_{64} & \mathrm{~m}_{65} & \mathrm{~m}_{66} & \mathrm{~m}_{67} & 0 \\
0 & 0 & \mathrm{~m}_{73} & \mathrm{~m}_{74} & \mathrm{~m}_{75} & \mathrm{~m}_{76} & \mathrm{~m}_{77} & 0 \\
0 & 0 & \mathrm{~m}_{83} & \mathrm{~m}_{84} & \mathrm{~m}_{85} & \mathrm{~m}_{86} & 0 & \mathrm{~m}_{88}
\end{array}\right),
$$

$$
\mathrm{V}(\mathrm{n})=\left(\begin{array}{c}
\mathrm{a}_{\mathrm{n}}^{(1)} \\
\mathrm{a}_{\mathrm{n}}^{(2)} \\
\mathrm{b}_{\mathrm{n}}^{(1)} \\
\mathrm{b}_{\mathrm{n}}^{(2)} \\
\mathrm{c}_{\mathrm{n}}^{(1)} \\
\mathrm{c}_{\mathrm{n}}^{(2)} \\
d_{\mathrm{n}}^{(1)} \\
d_{\mathrm{n}}^{(2)}
\end{array}\right), \quad \mathrm{F}(\mathrm{n})=\left(\begin{array}{c}
0 \\
0 \\
0 \\
0 \\
\mathrm{f}_{5}(\mathrm{n}) \\
\mathrm{f}_{6}(\mathrm{n}) \\
\mathrm{f}_{7}(\mathrm{n}) \\
\mathrm{f}_{8}(\mathrm{n})
\end{array}\right),
$$

$$
\begin{aligned}
& \mathrm{m}_{12}=\mathrm{g}_{\mathrm{n}}\left(\xi_{01}\right), \mathrm{m}_{13}=-\mathrm{g}_{\mathrm{n}}^{(1)}\left(\xi_{11}\right), \mathrm{m}_{14}=-\mathrm{g}_{\mathrm{n}}^{(1)}\left(\xi_{21}\right), \mathrm{m}_{15}=-\mathrm{g}_{\mathrm{n}}\left(\xi_{11}\right), \\
& \mathrm{m}_{16}=-\mathrm{g}_{\mathrm{n}}\left(\xi_{21}\right), \mathrm{m}_{21}=\mathrm{j}_{\mathrm{n}}\left(\xi_{01}\right), \mathrm{m}_{23}=\mathrm{q}_{1} \mathrm{~h}_{\mathrm{n}}^{(1)}\left(\xi_{11}\right), \mathrm{m}_{24}=\mathrm{q}_{2} \mathrm{~h}_{\mathrm{n}}^{(1)}\left(\xi_{21}\right), \\
& \mathrm{m}_{25}=\mathrm{q}_{1} \mathrm{j}_{\mathrm{n}}\left(\xi_{11}\right), \mathrm{m}_{26}=\mathrm{q}_{2} \mathrm{j}_{\mathrm{n}}\left(\xi_{21}\right), \mathrm{m}_{31}=\mathrm{H}_{0} \mathrm{~g}_{\mathrm{n}}\left(\xi_{01}\right) / \mathrm{E}_{0}, \\
& \mathrm{~m}_{33}=-\mathrm{p}_{1} \mathrm{~g}_{\mathrm{n}}^{(1)}\left(\xi_{11}\right), \mathrm{m}_{34}=-\mathrm{p}_{2} \mathrm{~g}_{\mathrm{n}}^{(1)}\left(\xi_{21}\right), \mathrm{m}_{35}=-\mathrm{p}_{1} \mathrm{~g}_{\mathrm{n}}\left(\xi_{11}\right), \\
& \mathrm{m}_{36}=-\mathrm{p}_{2} \mathrm{~g}_{\mathrm{n}}\left(\xi_{21}\right), \mathrm{m}_{42}=\mathrm{H}_{0} \mathrm{j}_{\mathrm{n}}\left(\xi_{01}\right) / \mathrm{E}_{0}, \mathrm{~m}_{43}=\mathrm{q}_{1} \mathrm{p}_{1} \mathrm{~h}_{\mathrm{n}}^{(1)}\left(\xi_{11}\right), \\
& \mathrm{m}_{44}=\mathrm{q}_{2} \mathrm{p}_{2} \mathrm{~h}_{\mathrm{n}}^{(1)}\left(\xi_{21}\right), \mathrm{m}_{45}=\mathrm{q}_{1} \mathrm{p}_{1} \mathrm{j}_{\mathrm{n}}\left(\xi_{11}\right), \mathrm{m}_{46}=\mathrm{q}_{2} \mathrm{p}_{2} \mathrm{j}_{\mathrm{n}}\left(\xi_{21}\right), \\
& \mathrm{m}_{53}=\mathrm{g}_{\mathrm{n}}^{(1)}\left(\xi_{12}\right), \mathrm{m}_{54}=\mathrm{g}_{\mathrm{n}}^{(1)}\left(\xi_{22}\right), \mathrm{m}_{55}=\mathrm{g}_{\mathrm{n}}\left(\xi_{12}\right), \mathrm{m}_{56}=\mathrm{g}_{\mathrm{n}}\left(\xi_{22}\right), \\
& \mathrm{m}_{58}=-\mathrm{g}_{\mathrm{n}}^{(1)}\left(\xi_{02}\right), \mathrm{m}_{63}=\mathrm{q}_{1} \mathrm{~h}_{\mathrm{n}}^{(1)}\left(\xi_{12}\right), \mathrm{m}_{64}=\mathrm{q}_{2} \mathrm{~h}_{\mathrm{n}}^{(1)}\left(\xi_{22}\right), \\
& \mathrm{m}_{65}=\mathrm{q}_{1} \mathrm{j}_{\mathrm{n}}\left(\xi_{12}\right), \mathrm{m}_{66}=\mathrm{q}_{2} \mathrm{j}_{\mathrm{n}}\left(\xi_{22}\right), \mathrm{m}_{67}=\mathrm{h}_{\mathrm{n}}^{(1)}\left(\xi_{02}\right), \\
& \mathrm{m}_{73}=\mathrm{p}_{1} \mathrm{~g}_{\mathrm{n}}^{(1)}\left(\xi_{12}\right), \mathrm{m}_{74}=\mathrm{p}_{2} \mathrm{~g}_{\mathrm{n}}^{(1)}\left(\xi_{22}\right), \mathrm{m}_{75}=\mathrm{p}_{1} \mathrm{~g}_{\mathrm{n}}\left(\xi_{12}\right), \\
& \mathrm{m}_{76}=\mathrm{p}_{2} \mathrm{~g}_{\mathrm{n}}\left(\xi_{22}\right), \mathrm{m}_{77}=-\mathrm{H}_{0} \mathrm{~g}_{\mathrm{n}}^{(1)}\left(\xi_{02}\right) / \mathrm{E}_{0},
\end{aligned}
$$




$$
\left.\begin{array}{l}
\mathrm{m}_{83}=\mathrm{q}_{1} \mathrm{p}_{1} \mathrm{~h}_{\mathrm{n}}^{(1)}\left(\xi_{12}\right), \mathrm{m}_{84}=\mathrm{q}_{2} \mathrm{p}_{2} \mathrm{~h}_{\mathrm{n}}^{(1)}\left(\xi_{22}\right), \mathrm{m}_{85}=\mathrm{q}_{1} \mathrm{p}_{1} \mathrm{j}_{\mathrm{n}}\left(\xi_{12}\right), \\
\mathrm{m}_{86}=\mathrm{q}_{2} \mathrm{p}_{2} \mathrm{j}_{\mathrm{n}}\left(\xi_{22}\right), \mathrm{m}_{88}=\mathrm{H}_{0} \mathrm{~h}_{\mathrm{n}}^{(1)}\left(\xi_{02}\right) / \mathrm{E}_{0}, \\
\mathrm{f}_{5}=\mathrm{E}_{0}^{(\mathrm{e})} \mathrm{A}_{\mathrm{n}}^{1}\left(\mathrm{k}_{0} \mathrm{~h}, 0\right) \mathrm{g}_{\mathrm{n}}\left(\xi_{02}\right) / \mathrm{E}_{0}, \mathrm{f}_{6}=-\mathrm{E}_{0}^{(\mathrm{m})} \mathrm{A}_{\mathrm{n}}^{1}\left(\mathrm{k}_{0} \mathrm{~h}, 0\right) \mathrm{j}_{\mathrm{n}}\left(\xi_{02}\right) / \mathrm{E}_{0}, \\
\mathrm{f}_{7}=\mathrm{H}_{0}^{(\mathrm{m})} \mathrm{A}_{\mathrm{n}}^{1}\left(\mathrm{k}_{0} \mathrm{~h}, 0\right) \mathrm{g}_{\mathrm{n}}\left(\xi_{02}\right) / \mathrm{E}_{0}, \mathrm{f}_{8}=-\mathrm{H}_{0}^{(\mathrm{e})} \mathrm{A}_{\mathrm{n}}^{1}\left(\mathrm{k}_{0} \mathrm{~h}, 0\right) \mathrm{j}_{\mathrm{n}}\left(\xi_{02}\right) / \mathrm{E}_{0}, \\
\xi_{01}=\mathrm{k}_{0} \mathrm{a}_{1}, \xi_{11}=\mathrm{k}_{1} \mathrm{a}_{1}, \xi_{21}=\mathrm{k}_{2} \mathrm{a}_{1}, \xi_{12}=\mathrm{k}_{1} \mathrm{a}_{2}, \xi_{22}=\mathrm{k}_{2} \mathrm{a}_{2}, \xi_{02}=\mathrm{k}_{0} \mathrm{a}_{2} .
\end{array}\right\}
$$

\section{Far field}

Using the asymptotic expression for the functions $\tilde{\overrightarrow{\mathrm{n}}}_{0 \mathrm{n}}\left(\mathrm{r}, \theta, \mathrm{k}_{0}\right)$, $\tilde{\overrightarrow{\mathrm{m}}}_{0 \mathrm{n}}\left(\mathrm{r}, \theta, \mathrm{k}_{0}\right)[21]$ :

$$
\left.\begin{array}{l}
\tilde{\overrightarrow{\mathrm{n}}}_{0 \mathrm{n}}\left(\mathrm{r}, \theta, \mathrm{k}_{0}\right) \approx(-\mathrm{i})^{\mathrm{n}} \frac{\mathrm{e}^{\mathrm{ik} \mathrm{k}_{0} \mathrm{r}}}{\mathrm{k}_{0} \mathrm{r}} \mathrm{P}_{\mathrm{n}}^{1}(\cos \theta) \overrightarrow{\mathrm{e}}_{\theta}, \\
\tilde{\overrightarrow{\mathrm{m}}}_{0 \mathrm{n}}\left(\mathrm{r}, \theta, \mathrm{k}_{0}\right) \approx-(-\mathrm{i})^{\mathrm{n}+1} \frac{\mathrm{e}^{\mathrm{ik} \mathrm{k}_{0} \mathrm{r}}}{\mathrm{k}_{0} \mathrm{r}} \mathrm{P}_{\mathrm{n}}^{1}(\cos \theta) \overrightarrow{\mathrm{e}}_{\varphi},
\end{array}\right\}
$$

we obtain the asymptotic representation of the electric vector $\overrightarrow{\mathrm{E}}_{2}$

$$
\overrightarrow{\mathrm{E}}_{2} \approx \mathrm{E}_{0} \frac{\mathrm{e}^{\mathrm{i} \mathrm{k}_{0} \mathrm{r}}}{\mathrm{k}_{0} \mathrm{r}} \vec{\Psi}(\theta), \mathrm{r} \rightarrow \infty
$$

where

$$
\left.\begin{array}{l}
\vec{\Psi}(\theta)=\Psi_{1}(\theta) \vec{e}_{\theta}+\Psi_{2}(\theta) \vec{e}_{\varphi}, \\
\Psi_{1}(\theta)=\sum_{n=1}^{\infty}(-i)^{n} P_{n}^{1}(\cos \theta) d_{n}^{(2)}, \quad \Psi_{2}(\theta)=-\sum_{n=1}^{\infty}(-i)^{n+1} P_{n}^{1}(\cos \theta) d_{n}^{(1)}
\end{array}\right\}
$$

The curve $D(\theta)=|\vec{\Psi}(\theta)|^{2}$ is a direction diagram of an electric field and characterizes the magnitude of the electromagnetic energy in the direction of $\theta$ :

$$
\mathrm{D}(\theta)=\left|\Psi_{1}(\theta)\right|^{2}+\left|\Psi_{2}(\theta)\right|^{2}
$$


We need to solve the system (20) in order to find the coefficients $d_{n}^{(1)}, d_{n}^{(2)}$ representation:

$$
\mathrm{d}_{\mathrm{n}}^{(1)}=\left|\mathrm{M}_{7}(\mathrm{n})\right| /|\mathrm{M}(\mathrm{n})|, \quad \mathrm{d}_{\mathrm{n}}^{(2)}=\left|\mathrm{M}_{8}(\mathrm{n})\right| /|\mathrm{M}(\mathrm{n})|,
$$

where $|M(n)|$ is a determinant of the matrix $M(n),\left|M_{j}(n)\right|$ is a determinant of the matrix $M_{j}(n), M_{j}(n)$ is a matrix $M(n)$, where $j$ column is replaced with a $\mathrm{F}(\mathrm{n})$ column-vector.

Using computer algebra system MathCAD [23, 24] the calculations of the direction diagram of an electric field were carried out for some parameters of the problem. The spherical functions were calculated by means of built-in functions. The derivatives of spherical functions were calculated by means of the formula [22]

$$
\frac{d}{d x} f_{n}(x)=n f_{n}(x) / x-f_{n+1}(x), n=0,1,2, \ldots .
$$

We assume that at the point $\mathrm{O}$ there is the electric dipole $\mathrm{H}_{0}^{(\mathrm{m})}=\mathrm{E}_{0}^{(\mathrm{m})}=0, \mathrm{E}_{0}^{(\mathrm{e})}=\mathrm{E}_{0}, \mathrm{H}_{0}^{(\mathrm{e})}=\mathrm{H}_{0}=\mathrm{E}_{0} \mathrm{k}_{0} / \mathrm{i} \omega \mu_{0}$ and the remaining parameters are equal: $\mathrm{a}_{1}=0.3 \mathrm{~m}, \mathrm{a}_{2}=0.35 \mathrm{~m}, \mathrm{~h}=0.7 \mathrm{~m}, \mathrm{f}=10^{9} \mathrm{~Hz}, \omega=2 \pi \mathrm{f}, \varepsilon=2.1 \varepsilon_{0}, \mu$ $=1,01 \mu_{0}$. Figure 2 shows the graphs of the direction diagram $D(\theta)$ for the increasing values of the Tellegen parameters $\tau=0,0.2 ; 0.4 ; 0.5 ; 0.6$ and $\mathrm{k}=0$. Figure 3 presents the graphs of the direction diagram $\mathrm{D}(\theta)$ of an electric field for the increasing values of the chirality parameters $\mathrm{k}=0,0.2 ; 0.4 ; 0.5 ; 0,6$ and $\tau$ $=0$. Figure 4 demonstrates the graphs of the direction diagram $\mathrm{D}(\theta)$ for the increasing values $\mathrm{a}_{1}=0.05,0.2,0.25,0.3$ and $\tau=0.8, \mathrm{k}=0.5, \mathrm{a}_{2}=0.35 \mathrm{~m}$.

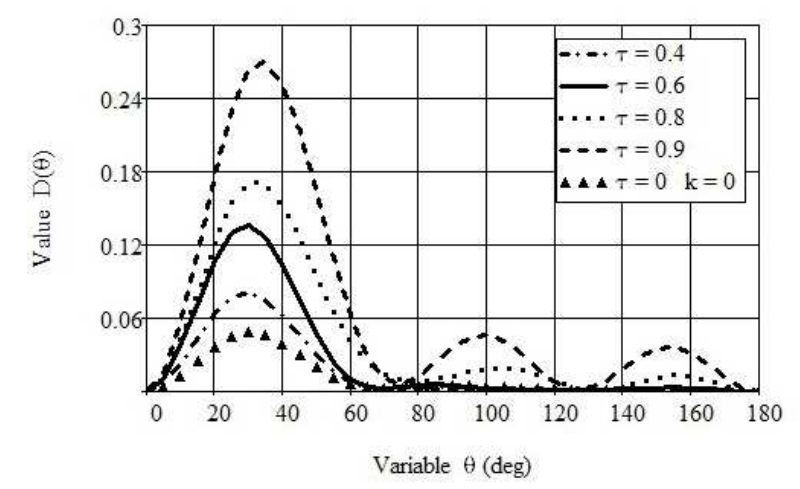

Fig. 2. Graphs of function $D(\theta)$ for some values of the Tellegen parameters $\tau$ and $\mathrm{k}=0$ 


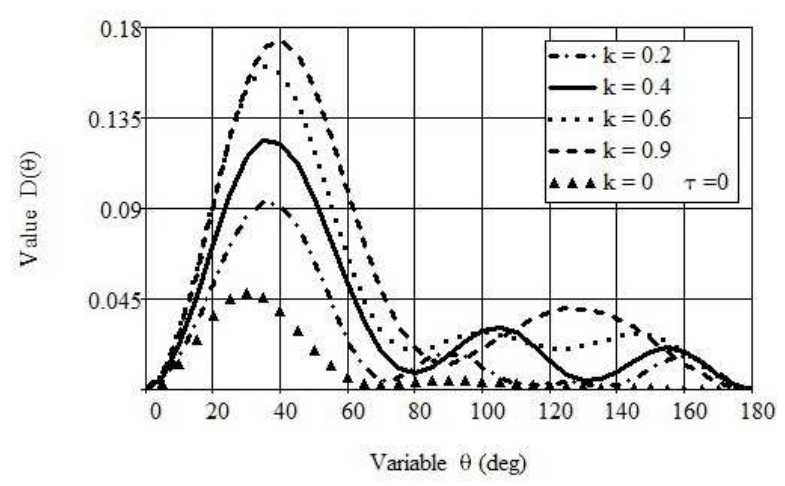

Fig. 3. Graphs of function $\mathrm{D}(\theta)$ for some values of the chirality parameters $\mathrm{k}$ and $\tau=0$

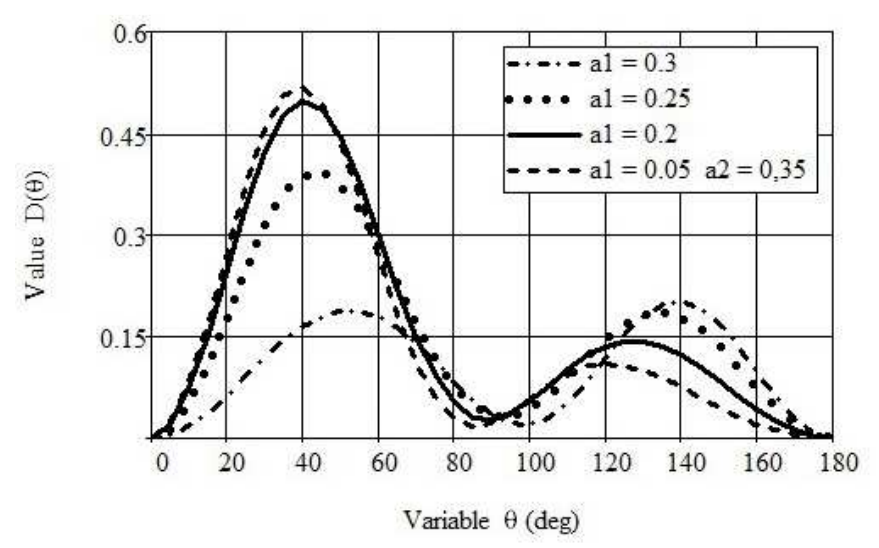

Fig. 4. Graphs of function $\mathrm{D}(\theta)$ for some values $\mathrm{a}_{1}$ and $\tau=0,8, \mathrm{k}=0,5, \mathrm{a}_{2}=0,35 \mathrm{~m}$.

\section{Conclusions}

The paper developed analytical algorithm for solving an axisymmetric problem of diffraction of electromagnetic field of electric and magnetic dipoles on the bi-isotropic spherical shell. The solution of the problem is reduced to solving a system of linear algebraic equations. The developed method can find practical application in the development and design of electromagnetic screens. The shielding of electromagnetic fields, as a basis for environmental security, allows to complete the following tasks - protection of information leakage through technical channels; electromagnetic compatibility of equipment and devices when they are used together; people protection from the increased level of electromagnetic fields and creation of a favorable environment around operating electric and microwave devices. 


\section{Acknowledgement}

The research leading to these results has supported by funding from the People Programme (Marie Curie International Research Staff Exchange) of the EU FP7/20072013/ under REA grant agreement n PIRSES-GA-2013-610547

\section{References}

[1] Lakhtakia A., Varadan V.K., Varadan V.V.: Time-harmonic electromagnetic fields in chiral media. Lecture Notes in Physics, Springer, 1989.

[2] Cui Tie Jun, Smith D.R., Lui R.: Metamaterials. Theory, Design and Applications, Springer, 2009.

[3] Vendik I. B. Vendik O. G.: Metamaterials and their application in microwaves: A review. Tech. Phys., 1 (2013), 1-24.

[4] Lindell I.V., Sihvola A.H., Tretyakov S.A., Viitanen A.J.: Electromagnetic waves in chiral and bi-isotropic media. London, Artech House, 1994.

[5] Ivanov O.V.: Electromagnetic wave propagation in anisotropic and bianisotropic layered structures, Ulyanovsk STU, 2010.

[6] Behaviour of Electromagnetic Waves in Different Media and Structures. Ed. by A.Akdagli. InTech, 2011.

[7] Neganov V.A., Osipov O.V.: Approximate boundary conditions for a thin chiral layer located on a perfectly conducting plane, J. commun. Tech. and electr., 3 (2005), 270-275.

[8] Sirenko Y, Velychko L.: Electromagnetic waves in complex systems. Selected Theoretical and applied problems, Springer, 2016.

[9] Shorohova E.: Diffraction of electromagnetic waves on chiral bodies of rotation. Analytical approach. Saarbruchen, LAP LAMBERT Academic Publishing, 2013.

[10]Solutions and Applications of Scattering, Propagation, Radiation and Emission of Electromagnetic Waves. Ed. A. Kishk. InTech, 2912.

[11]Monakhov M., Shorohova E.: Radiant properties of antennas in composite media. Metamaterials and chiral media. Saarbruchen, LAP LAMBERT Academic Publishing, 2011.

[12]Kapshai V.N., Kondratyuk V.V.: The scattering of electromagnetic waves by bi- isotropic ball in an isotropic medium, Prob. Phys., Math. Tech., 3(2010), 17-21.

[13]Belichenko V.I., Fisanov V.V.: Scattering of electromagnetic waves by bi-isotropic sphere. News high schools. Physics, 10(1994), 108-112.

[14]Erofeenko V.T., Malyi S.V.: Diffraction of a plane electromagnetic wave by a flat layer of bi-isotropic material. Vestnik BSU. Ser.1, 2(2010), 11-16.

[15]Erofeenko V.T., Bondarenko V.F Numerical study of the interaction of electromagnetic fields, electric and magnetic dipoles with a composite screen. Izv. NAN Belar. Ser. Fiz.- mat. n., 4(2013), 113-120.

[16]Neganov V.A., Osipov O.V.: Reflection of electromagnetic waves from the plane of chiral structures. Izv. Vuz. Radiofizika, 9(1999), 870-878.

[17]Neganov V.A., Osipov O.V.: Features the reflection of electromagnetic waves from the planar chiral structures. Phys. wave proc. and radio systems, 1(1999), 5-11. 
[18]Neganov V.A., Osipov O.V.: Scattering of plane electromagnetic waves on chiral metal cylinder. Tech. Phys. Letters, 1(2000), 77-83.

[19]Ivanov E. A.: Diffraction of electromagnetic waves on two bodies, Springfield, Washington 1970.

[20] Shushkevich G.Ch., Kuts A.I.: Numerical investigation of scattering field of electrical dipole on the bi-isotropic ball, Informatika, 2 (2015), 46-54.

[21]Erofeenko V.T.: Analytical modelling in the electrodynamics, CD LIBROKOM, Moscow 1989.

[22]Handbook of Mathematical Functions: with Formulas, Graphs and Mathematical Tables. Eds. by M. Abramowitz and I. A. Stegun. Dover, New York, 1972.

[23]Shushkevich G.Ch., Shushkevich S.V.: Computer technology in mathematics. The system Mathcad 14: in 2 parts. Grevsova, Minsk 2012.

[24]Fausett L.V.: Numerical Methods Using MathCAD. Prentice Hall, New Jork 2002.

\section{ROZPRASZANIE FAL ELEKTROMAGNETYCZNYCH ZA POMOCA BIIZOTROPOWEJ POWŁKI KULISTEJ}

\section{Streszczen i e}

W artykule przedstawiono analityczne rozwiązanie problemu rozpraszania fal elektromagnetycznych na biizotropowej powłoce kulistej. Wtórne fale elektromagnetyczne są przedstawiane jako superpozycja funkcji falowych wektora kulistego. Rozwiązanie problemu brzegowego zostało zredukowane do rozwiązania układu liniowych równań algebraicznych dla współczynników, które są zawarte w opisie pól wtórnych. Otrzymano wzór do obliczania diagramu kierunkowego pola elektrycznego w części pola oddalonej na dużą odległość od źródła promieniowania oraz otrzymano wyniki liczbowe dla różnych wartości parametrów. Wyniki mogą znaleźć praktyczne zastosowanie w rozwoju i projektowaniu ekranów elektromagnetycznych.

Słowa kluczowe: biizotropowe powłoki kuliste, fale elektromagnetyczne, funkcje falowe wektora kulistego, diagram kierunkowy pola elektrycznego

DOI: $10.7862 / \mathrm{rm} .2017 .09$

Otrzymano/received: 12.07.2016

Zaakceptowano/accepted: 24.11 .2016 